\section{WIENER KLINISCHE} WOCHENSCHRIFT

The Middle European Journal of Medicine

Printed in Austria

(c) Springer-Verlag 2006

\section{Akute infektiöse Diarrhoe}

\section{Gertrude Seiberl, Elisabeth Bischof und Christoph Wenisch}

4. Medizinische Abteilung mit Infektions- und Tropenmedizin, SMZ-Süd, Kaiser Franz Josef Spital, Wien, Österreich
In der Anamnese finden sich wichtige Hinweise auf den Krankheitserreger

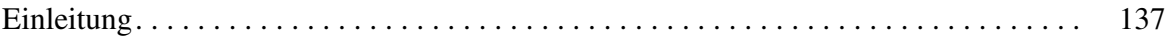

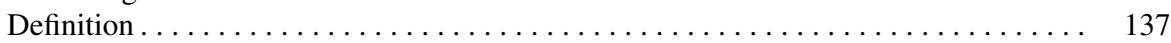

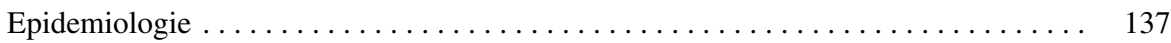

Management von Patienten mit akuter Diarrhoe . . . . . . . . . . . . . . . . . . . 138

Diagnostik . . . . . . . . . . . . . . . . . . . . . . . . . . . . . . . . . . . . 139

Ia) Akute virale Diarrhoe . . . . . . . . . . . . . . . . . . . . . . . . . . . . . . . 140

Ib) Akute bakterielle Diarrhoe . . . . . . . . . . . . . . . . . . . . . . . . . . . . 142

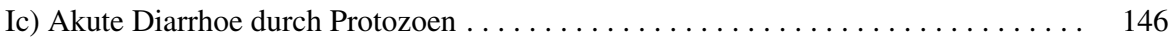

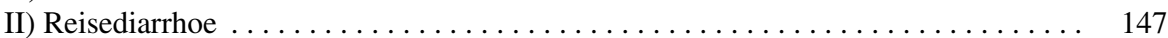

III) Nosokomiale Diarrhoe. . . . . . . . . . . . . . . . . . . . . . . . . . . . . . . . 148

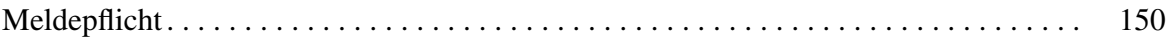

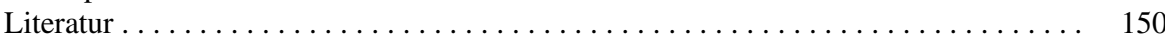

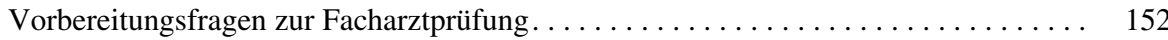

\section{Einleitung}

Infektiöse Durchfallserkrankungen sind weltweit die zweithäufigste Krankheits- und Todesursache mit regionalen Unterschieden in der Prävalenz der verursachenden Erreger [1]. In Industrieländern treten im Schnitt 1,4 Durchfallsepisoden pro Erwachsenem und Jahr auf. Kinder unter 3 Jahren erkranken vor allem im Winter doppelt so häufig [2]. In Entwicklungsländern sind Durchfallserkrankungen die häufigste Ursache für Tod im Kindesalter und führen in der Folge von Mangelernährung zu verzögerter körperlicher und intellektueller Entwicklung.

\section{Definition}

Unter akuter Diarrhoe versteht man das Absetzen von mehr als 3 breiigen/flüssigen Stühlen pro Tag bzw. ein Überschreiten des Stuhlgewichtes von 200 g/Tag. Man unterscheidet:

- akute Diarrhoe: Dauer < 14 Tage,

- persistierende Diarrhoe: Dauer $>14$ Tage,

- chronische Diarrhoe: Dauer $>30$ Tage.

Mehr als die Hälfte der Erkrankungen sind selbstlimitierend und von kurzer Dauer ( $<24$ Stunden).

\section{Epidemiologie}

Die akute infektiöse Diarrhoe wird unterschieden in:

a) Ambulant erworbene akute Diarrhoe - vorwiegend verursacht durch:

- virale Erreger: Noroviren, Adenoviren, Rotaviren - sie treten gehäuft in den Wintermonaten auf;

- Bakterien: Salmonella spp., Campylobacter spp., Shigella spp., Yersinia spp., E. coli spp.;

- toxininduzierte Diarrhoe: S. aureus, Bacillus cereus.

b) Reisediarrhoe

Das Risiko an einer Reisediarrhoe zu erkranken hängt ab vom:

- Reiseland: Länder mit niedrigem Risiko $(<10 \%)$ sind Nordeuropa, Kanada, Vereinigte Staaten, Australien, Neuseeland; Länder mit hohem Risiko (>30\%) sind Staaten in Afrika (außer Südafrika), in Asien, Mittel- und Südamerika;

- Reisestil: betroffen sind vor allem Individual- und Abenteuerreisende;

- Hygieneverhalten: die Expositionsprophylaxe führt zu einer deutlichen Risikoreduktion; 
Management des Patienten anhand von Epidemiologie und Klinik

Epidemiologie
- individueller Disposition des Reisenden: z.B. Alter, Grundkrankheiten.

Die Erreger der Reisediarrhoe sind sowohl bakteriell (Salmonella und Campylobacter spp., E. coli [ETEC], Shigella und Vibrio spp.), viral (Rota- und Noroviren) als parasitär (Entamöba histolytica, Giardia lamblia, Cyclospora, Cryptosporidium).

\section{c) Nosokomiale Diarrhoe}

Diese tritt nach $>3$ Tagen Spitalsaufenthalt auf und wird in erster Linie verursacht durch Clostridium difficile (vorausgehende Antibiotikatherapie!) und Noroviren.

Giardia lamblia, Cryptosporidium, Cyclospora kommen auch als Erreger einer persistierenden Diarrhoe in Betracht. Handelt es sich um immunkompromitierte Patienten, sind M. avium complex und Mikrosporidia in die Diagnostik einer akuten oder persistierenden Diarrhoe mit einzubeziehen.

\section{Management von Patienten mit akuter Diarrhoe}

Ein Algorithmus zum Vorgehen bei Patienten mit akuter Diarrhoe ist in Tabelle 1. angeführt.

Aus Epidemiologie und Klinik können wesentliche Hinweise auf den wahrscheinlichen Erreger gewonnen werden und die weiteren diagnostischen Maßnahmen gesetzt und nach einer symptomatischen Therapie auch eine allenfalls notwendige gezielte Antibiotikatherapie begonnen werden.

\section{a) Epidemiologische Faktoren}

Reiseanamnese, Speisenanamnese (Eier, rohes Fleisch, Geflügel, Meeresfrüchte, unpasteurisierte Milch, verdorbene Nahrungsmittel), Kontakt zu Erkrankten, Arbeit in einer Betreuungsorganisation (Tageszentrum, Pflegeheim, Kindergarten), vorausgehende Antibiotikatherapie, Tätigkeit im Lebensmittelbereich, zugrunde liegende Erkrankungen oder Dispositionen (AIDS, immunsuppressive Therapie, St. p. Gastrektomie, höheres Lebensalter).

\section{Tabelle 1}

\section{Management von Patienten mit akuter Diarrhoe}

Evaluieren der Schwere der Erkrankung

Ausmaß des Dehydrierung, \pm Fieber, Krämpfe, Dauer und Intensität der Diarrhoe

$\downarrow$

Symptomatische Therapie

Rehydratation p.o. / i.v., Spasmolytika

$\downarrow$

Evaluierung der Epidemiologie

$\downarrow$

Stuhlmikroskopie

Invasive / nicht invasive Stühle

$\downarrow$

\begin{tabular}{|c|}
\hline $\begin{array}{c}\text { Ausserhalb des Krankenhauses } \\
\text { erworben Salmonellen, Shigellen, } \\
\text { Campylobacter, E. coli O157: H7, } \\
\text { C.difficile Toxin A (+B) }\end{array}$ \\
\begin{tabular}{|c|} 
(>3 Tage Krankenhausaufenthalt) \\
C. difficile Toxinnachweis
\end{tabular} \\
\hline Antibiotikatherapie \\
\hline
\end{tabular}




\begin{tabular}{|lll|}
\hline Tabelle 2 & & \\
\hline \multicolumn{2}{|l|}{ Zusammenhang zwischen } & Klinik, Inkubationszeit und Erreger \\
\hline Vorwiegendes Symptom & Erreger & $I K Z$ \\
\hline Erbrechen & S. aureus & $1-6 \mathrm{Std}$ \\
& B. cereus & $1-6 \mathrm{Std}$ \\
& Noroviren & $24-48$ Std \\
Wässrige Diarrhoe & ETEC & $1-3$ Tage \\
& C. perfringens & $8-16$ Std \\
& C. parvum & $2-28$ Tage \\
& Campylobacter & $2-5$ Tage \\
Invasive Diarrhoe & Salmonellen (ohne Typhus) & $1-3$ Tage \\
& Shiga.Toxin prod. E.coli & $1-8$ Tage \\
& Shigellen & $1-3$ Tage \\
\hline
\end{tabular}

\section{b) Klinische Faktoren}

- Allmählicher oder plötzlicher Beginn der Erkrankung, Dauer der Symptome, Stuhlfrequenz und relative Menge, \pm Fieber, Krämpfe, Stuhlbeschaffenheit (wässrig, breiig, schleimig, blutig, eitrig);

- Begleitsymptome wie Übelkeit, Erbrechen, Kopfschmerzen, Muskelschwäche und -krämpfe, Verlangsamung;

- Symptome des Volumenmangels wie Tachykardie, Orthostase, Einschränkung der Harnmenge, verminderter Hautturgor;

- Zusammenhang zwischen Klinik, Inkubationszeit und Erreger (Tabelle 2).

\section{Diagnostik}

Stuhlmikroskopie

Diese führt zur Diagnose einer invasiven Diarrhoe durch den Nachweis von Leukozyten und Erythrozyten im Stuhl, mit einer Sensitivität und Spezifität für den Nachweis von Leukozyten von 73-84\% [3]; ein positiver Laktoferrintest kann diese Diagnose unterstützen. Laktoferrin ist ein Marker für fäkale Leukozyten, der Test besitzt eine Sensitivität und Spezifität zwischen 90 und 100\% [4], kann jedoch nicht unterscheiden zwischen infektiöser und nicht-infektiöser Diarrhoe, sondern ist bei allen entzündlichen Darmprozessen, z.B. auch bei chronisch entzündlichen Darmerkrankungen, positiv.

\section{Stuhlkultur}

Routinestuhlkulturen ohne entsprechende Epidemiologie und Klinik einer schweren Erkrankung (Fieber, krampfartige Bauchschmerzen, invasive Stühle, Zeichen der Dehydratation und Elektolytentgleisung) führen in einem enttäuschend niedrigem Prozentsatz (1,5-5,6\%) [5] zur Diagnose.

Trotz dieser Limitationen sollten Stuhlkulturen bei Patienten mit passender Epidemiologie und Klinik sowie bei folgenden Patienten gewonnen werden:

- immunkompromittierte inkl. HIV-positive Patienten,

- Patienten mit Begleiterkrankungen, die das Risiko von Komplikationen erhöhen (Aneurysmen, chronisch entzündliche Darmerkrankung, Diabetes mellitus),

- Patienten, die im Lebensmittelbereich, Pflegebereich, Betreuungsinstitutionen arbeiten und zur Arbeit zurückkehren sollen.

\section{Endoskopie}

Sie ist in der Diagnostik der akuten Diarrhoe nicht indiziert, dient nach Vorliegen von negativen Stuhlkulturen, weiterbestehender Klinik dann aber zur Differentialdi- 
agnose u.a. der chronisch entzündlichen Darmerkrankung durch Biopsie und Histologie. Weiters zeigt sie bei einer Clostridium difficile Toxin verursachten Diarrhoe das typische Bild der pseudomembranösen Kolitis, ist für deren Diagnose jedoch nicht nötig, da dies durch Toxinnachweis und Kultur erfolgt.

\section{Parasitologische Stuhluntersuchungen}

Diese sind indiziert bei:

- einer Reisediarrhoe und negativen Stuhlkulturen,

- einer persistierenden Diarrhoe (Gardia lamblia, Cryptosoridium, Entamoebea histolytika, Cyclspora),

- HIV-positiven Patienten,

- bei Colitispatienten, vor geplanter Cortisontherapie,

- einer blutigen Diarrhoe mit wenigen oder keinen fäkalen Leukozyten (Verdacht auf intestinale Amöbiasis),

- nach negativem E. coli-Nachweis (ETEC).

Stellenwert von viralen Gastroenteritiden

Epidemiologie

\section{Ia) Akute virale Diarrhoe \\ Epidemiologie}

Viren sind die häufigsten Erreger von Durchfallserkrankungen in Industriestaaten. Den Aufzeichnungen Europäischer Surveillance Systemen zufolge werden 85\% aller Gastroenteritisausbrüche von Viren verursacht [6].

Die vier häufigsten viralen Erreger von Magen-Darm-Infektionen sind Noroviren, Rotaviren, Adenoviren und Astroviren. Andere virale Erreger wie Coronavirus, Aichi Virus, Picornavirus und Pestivirus haben keine praktische Bedeutung. Allerdings gelingt nur in 1/3 aller suspizierten Fälle von viraler Gastroenteritis ein Erregernachweis, was die Existenz von weiteren bis jetzt unbekannten Viren wahrscheinlich macht.

Um virale Gastroenteritisausbrüche rasch von bakteriellen unterscheiden zu können, wurden 1982 von J. E. Kaplan klinische Kriterien etabliert, die bis heute Anwendung finden und hoch spezifisch sind [7]. Liegt demnach bei mehr als der Hälfte der Betroffenen Erbrechen vor, eine Inkubationszeit von 24 bis 48 Stunden, eine Krankheitsdauer von 12 bis 60 Stunden, sowie kein Nachweis eines bakteriellen Erregers in der Stuhlkultur, kann von einer viralen Genese der Durchfallserkrankung ausgegangen werden.

\section{Norovirus}

Bereits 1929 beschrieb J. Zahorsky erstmals eine „winter vomiting disease“ [8]. Es dauerte allerdings noch fast 50 Jahre, bis 1972 das Norovirus (früher nach dem Ort der Erstbeschreibung Norwalk/Ohio Virus genannt) als auslösendes Agens dieses bekannten Krankheitsbildes elektronenmikroskopisch nachgewiesen wurde. Heute weiß man, dass Noroviren die häufigsten Erreger von akuten Magen-Darm-Entzündungen sind und für den Großteil der viralen Gastroenteritiden bei Kindern (30\%) und bei Erwachsenen (bis zu 50\%) verantwortlich sind.

Noroviren sind kleine $30 \mathrm{~nm}$ große hüllenlose RNA-Viren. Aufgrund ihrer Genomorganisation werden sie der Familie der Caliciviridae zugeordnet. Sie werden in 5 Genogruppen (I, II und IV humanpathogen) unterteilt, innerhalb derer man verschiedene Genotypen unterscheidet.

Noroviren sind weltweit verbreitet und kommen bei Menschen aller Altersgruppen vor. Verursacht durch die hohe Infektiosität und Befallsrate ist ein epidemisches Auftreten in Gemeinschaftseinrichtungen, Kinder- und Altenheimen sowie Krankenhäusern charakteristisch. Weitere große Ausbrüche wurden auf Kreuzfahrtschiffen, in Militäreinheiten und Sportteams beschrieben. In den letzten Jahren ist es in Europa zu einem deutlichen Anstieg an Infektionen mit einer neuen Virusvariante (BristolVirus) gekommen [9].

Noroviren werden in großen Mengen über den Stuhl und Erbrochenes ausgeschieden. Übertragen wird das Virus in erster Linie auf fäkal-oralem Weg direkt von Mensch zu Mensch oder indirekt über kontaminierte Nahrungsmittel und Wasser. Allerdings kann auch die Inhalation von virushältigen Aerosolen zur Ansteckung führen. Die 
minimale Infektionsdosis ist äußerst gering und liegt bei 10-100 Viruspartikeln. Ansteckungsfähigkeit besteht zumindest ab dem Beginn der akuten Erkrankung bis 48 Stunden nach Ende der Symptome. Eine asymptmatische Virusausscheidung wurde jedoch auch prodromal sowie bis 21 Tage nach Krankheitsbeginn nachgewiesen. Klinisch präsentieren sich Betroffene nach einer Inkubationszeit von 24 bis 48 Stunden meist mit plötzlich einsetzender Übelkeit, Erbrechen und Durchfall. Dazu kommen Kopfschmerzen, Myalgien, subfebrile Temperaturen sowie allgemeines Krankheitsgefühl. Bereits nach 12 bis 72 Stunden ist der Großteil der Patienten wieder beschwerdefrei. Die genaue Pathogenese von norovirusinduzierter Diarrhoe ist bis heute nicht geklärt. Histologisch kommt es zur reversiblen entzündlichen Veränderungen der Jejunalschleimhaut mit Verletzung der Mikrovilli.

Aufgrund der typischen Symptomatik wird die Diagnose meist klinisch gestellt. Golden Standard der Diagnostik ist heute der Virusnachweis aus dem Stuhl mittels RT-PCR. Nur in speziellen Settings kommt noch die Elektronenmikroskopie zum Einsatz.

Noroviren sind extrem stabil und infektiös. Besonders bei Ausbrüchen in Spitälern oder Betreuungseinrichtungen müssen daher spezielle Hygienemaßnahmen (Isolierung von Erkrankten, Händedesinfektion, Mundschutz) zur Unterbrechung der Infektionskette eingehalten werden. Erkranktes Personal muss mindestens 48 Stunden nach Ende der Symptomatik freigestellt werden.

\section{Rotavirus}

Rotaviren sind unbehüllte $75 \mathrm{~nm}$ große Viruspartikel und gehören zur Familie der Reoviridae. Man unterscheidet 7 Serogruppen (A-G), wobei Rotaviren der Gruppe A weltweit am bedeutendsten sind. Anhand ihrer Oberflächenproteine (VP4 und VP7) können außerdem verschiedene Genotypen unterschieden werden.

Von Rotavirusinfektionen betroffen sind in erster Linie Säuglinge und Kleinkinder im Alter von 6 Monaten bis 2 Jahren. In dieser Patientengruppe sind Rotaviren die häufigste Ursache von Darminfektionen und tragen in den Entwicklungsländern aufgrund der fehlenden Therapiemöglichkeiten maßgeblich zur hohen Mortalität im Kindesalter bei. Die Bedeutung von Rotavirusinfektionen bei Erwachsenen wurde lange Zeit unterschätzt, allerdings verläuft die Erkrankung meist milder oder symptomlos [10].

Die Übertragung der Viren erfolgt hauptsächlich durch Schmierinfektion, kann aber auch durch kontaminiertes Wasser und Lebensmittel oder aerogen erfolgen. Bereits 10-100 Viruspartikel führen bei Kindern zur Infektion. Nach einer Inkubationszeit von 1 bis 3 Tagen kommt es dann zur Erkrankung mit Erbrechen, wässriger Diarrhoe, Fieber und Bauchschmerzen. In 30-50\% der Fälle bestehen zusätzlich respiratorische Symptome. Pathogenetisch kommt es zur Entzündung der Dünndarmmukosa mit Verlust von Mikrovilli. Daraus resultiert die Malabsorption und osmotische Diarrhoe. Direkt toxische Effekte auf die Schleimhautzellen dürfte das NSP4 Protein des Rotavirus haben [11]. Besonders bei Säuglingen ist die Gefahr der Dehydratation groß, rasche Flüssigkeitssubstitution ist daher die wichtigste Maßnahme. Komplizierend auftreten können Dünndarminvaginationen, Krampfanfälle und Enzephalopathie sowie nekrotisierende Enterokolitiden. Der Virusnachweis aus dem Stuhl erfolgt heute routinemäßig durch Immunassays (ELISA) oder PCR. Elektronenmikroskopie und Virusanzüchtung in der Zellkultur werden selten durchgeführt. Serologische Methoden haben keine Bedeutung für die Diagnostik.

\section{Adenovirus}

Adenoviren vom Serotyp 40 und 41 verursachen $10 \%$ aller pädiatrischen Gastroenteritiden in gemäßigten Klimazonen. Die Inkubationszeit ist mit 8-10 Tagen deutlich länger als bei Rota- und Norovirusinfektionen.

\section{Astrovirus}

Astroviruserkrankungen versuchen 3-9\% aller endemischen Diarrhoen bei Kindern und sind häufige nosokomiale Infektionen. Betroffen sind auch ältere Patienten in Pflegeeinrichtungen sowie Immunkompromittierte. Die Übertragung erfolgt fäko-oral und über kontaminierte Nahrungsmittel und Wasser.
Klinik der Norovirus assoziiierten Diarrhoe

Cave! - höchst infektiös

Übertragung und Klinik der Rotaviruserkrankung 


\section{Prävention}

Virale Gastroenteritiden stellen weltweit ein bedeutendes Gesundheitsproblem dar und sind in den Industriestaaten mit einer beträchtlichen Zahl an Hospitalisierungen und Arztkontakten verbunden. In Ländern der 3. Welt dagegen sterben täglich 1500 Kinder an Rotavirusinfektionen. 1998 wurde erstmals in den USA ein Rotavirusimpfstoff zugelassen, der jedoch bereits 1999 aufgrund von gehäuft aufgetretenen Dünndarminvaginationen wieder vom Markt genommen werden musste. Derzeit sind 2 orale Rotavirusvakzine in einigen Ländern bereits zugelassen und könnten einen großen Betrag zur Reduktion der Kindersterblichkeit leisten [12]. Impfstoffe gegen Norovirusinfektionen sind derzeit noch in Entwicklung.

\section{Ib) Akute bakterielle Diarrhoe}

\section{Pathophysiologie}

Bakterielle Erreger von Darminfektionen verfügen über einen oder mehrere Pathogenitätsmechanismen

a) Adhärenz: spezielle Eiweißstrukturen der Bakterienoberfläche verhindern die mechanische Entfernung durch die Darmperistaltik und so können zusätzlich produzierte Enterotoxine (z.B. des ETEC - entertoxinproduzierender E. coli) nahe an die Darmepithelien gebracht werden.

Pathogenitätsmechanismen der bakteriellen Erreger b) Toxine: durch Steigerung der intrazellulären cAMP und GMP kommt es zur massiven aktiven Sekretion von Elektrolyten und Wasser in das Darmlumen; Zytotoxine wie das Shiga-Toxin der Shigella dysenteriae oder das Shiga-like-Toxin des enterohämorrhagischen E. coli (EHEC) führen zu Zelltod in Gewebekulturen, aber auch zu Schäden an entfernten Organsystemen (z.B. hämolytisch-urämisches Syndrom des EHEC). Toxine von Clostridium botulinum, S. aureus (Enterotoxin B) und von Bacillus cereus sind Neurotoxine.

c) Invasion: Bakterien dringen in die Epithelzellen der Darmmukosa ein, vermehren sich dort und führen zum Tod der Wirtszelle. Dies ist von einer massiven inflammatorischen Reaktion begleitet, in die Entzündungsmediatoren wie Interleukine, Leukotriene und andere vasoaktive Stoffe involviert sind. Klinische Zeichen für dieses Geschehen sind Fieber, Krämpfe, häufige geringvolumige Stühle, auch schleimigeitrige, blutige Beimengungen.

Erregerspezifischer Wirkort ist der Dünn- und Dickdarm (Tabelle 3).

\begin{tabular}{|lll|}
\hline Tabelle 3 & & \\
\hline Stuhlmikroskopie und Erreger-spezifischer Wirkort in Dünn- und Dickdarm \\
\hline Erreger & $\begin{array}{l}\text { Nicht-invasive Diarrhoe } \\
(\text { Dünndarm })\end{array}$ & $\begin{array}{l}\text { Invasive Diarrhoe } \\
(\text { Dickdarm })\end{array}$ \\
\hline Bakterien & Salmonellen ${ }^{1}$ & Campylobacter ${ }^{1}$ \\
& E.coli - ausser EIEC & Shigellen \\
& C. perfringens & C.difficile \\
& S. aureus & Yersinien \\
& B. cereus & EIEC (entero invasive E. coli) \\
Vibrio cholerae & \\
Viren & Noroviren & Adenoviren \\
& Rotaviren & CMV, HSV \\
Protozoen & Giardia lamblia & Entamoeba histolytica \\
& Cryptosporidien & \\
Cyclospora & \\
\hline${ }^{1}$ Kann Dünn- und Dickdarm involvieren.
\end{tabular}




\section{Salmonellen}

sind gramnegative, bewegliche, fakultativ anaerobe Bakterien. Sie gehören zu einer einzigen Spezies: Salmonella cholerasuis, und werden durch O-, H- und Vi-Antigene subtypisiert.

Krankheitsbilder, die von Salmonellen verursacht werden sind:

1) akute Gastroenteritis: Am häufigsten werden S. enteritidis und S. typhimurium als Erreger isoliert.

Erregerreservoir sind Tiere (insbesondere Geflügel) und der Mensch (der Erkrankte bzw. der Dauerausscheider infolge von mangelnder Hygiene). Nahrungsmittel wie Eier, Fleisch müssen ausreichend gegart werden; Vorsicht ist geboten bei rohen oder weich gekochten Eiern und bei Fleisch, das nicht durch gebraten ist.

Eine Erkrankung entwickelt sich bei ausreichender Keimbelastung und reduzierter Abwehr:

- Keimbelastung bzw. Infektionsdosis: $\geq 10^{3}$,

- reduzierte Abwehr: erniedrigte Magensäure z. B. nach Gastrektomie oder durch $\mathrm{H}_{2}$-Blocker-Therapie [13].

Die Inkubationszeit beträgt 1-3 Tage.

2) Staus febrilis: durch S. typhi und S. paratyphi A, B und C.

In unseren Regionen sind Typhus abdominalis und Paratyphus typische Reise-assoziierte Erkrankungen und sind keine akuten Durchfallserkrankungen. Erregerreservoir sind kontaminiertes Wasser und Lebensmittel. Die Infektionsdosis beträgt $\geq 10^{2} \mathrm{Er}-$ reger.

Nach Aufnahme in den Organismus penetrieren die Bakterien die Dünndarmmukosa und gelangen über die intestinalen Lymphfollikel in die retikuloendothelialen Zellen von Leber und Milz. Diese Phase ist klinisch asymptomatisch und dauert 7-14 Tage. Anschließend gelangen sie in den Blutstrom und verursachen so die typischen Symptome von Typhus und Paratyphus:

Fieber, Schüttelfrost, Kopfschmerzen, Übelkeit, Erbrechen, trockenen Reizhusten, Myalgien, Verlangsamung, Verwirrung, Bradykardie, selten Diarrhoe (bei HIV positiven Patienten) - eher Verstopfung. Die sogenannten Typhusroseolen (2-4 mm große, hellrote makulopapulöse Läsionen vorwiegend im Bauch- und Brustbereich) sind selten zu beobachten; aus ihnen kann der Erregernachweis ebenfalls geführt werden.

Die Laborparameter zeigen eine Leukopenie mit Linksverschiebung, \pm Thrombopenie, mäßige Erhöhung der Transaminasen (LDH).

Die Diagnose wird gesichert durch den Nachweis von S. typhi/paratyphi in der Blutkultur (positiv in 60-80\%). Stuhlkulturen sind nicht geeignet zur Diagnostik (positiv in $30 \%$ ). Kulturen von Knochenmarkspunktaten sind in höherem Prozentsatz positiv als Blutkulturen (80-95\%), stellen jedoch einen nach Erheben einer genauen Anamnese und Klinik nicht notwendigen invasiven Eingriff dar. Die serologische Untersuchung (Widal-Test) ist für Diagnose der akuten Erkrankung nicht geeignet [14]. Die Komplikationen sind: Perforation, Blutung, DIC, Schock.

3) Bakteriämie: mit konsekutiver Osteomyelitis, Abszedierung (Milzabszess), Ausbildung eines mykotischen Aneurysmas oder einer septischen Arthritis.

HLA B27 positive Menschen neigen zur Entwicklung einer reaktiven Arthritis im Anschluss an eine Salmonellose.

4) Dauerausscheider: Wenn nach einer akuten Gastroenteritis länger als 6 Monate Salmonellen im Stuhl nachgewiesen werden, ohne dass Krankheitssymptome bestehen, spricht man von einem Dauerausscheider (entwickelt sich in 1-4\% der Fälle). Von Bedeutung ist das für Personen, die im Lebensmittelbereich, Pflegebereich u.ä. arbeiten.

Eine Sanierung von Dauerausscheidern kann erfolgen durch Gabe von Ciprofloxacin (500 mg b.i.d. über 2 bis 4 Wochen) \pm durch Sanierung der Gallenwege, da häufig Erkrankungen der Gallenwege (Gallensteine) vorliegen.

Krankheitsbilder, die von Salmonellen spp. verursacht werden

Typhus ist keine Durchfallserkrankung

Definition des

Dauerausscheiders 
Campylobacter: Fieber, Tenesmen, blutige Diarrhoe

Cave! - Komplikationen der Shigellose

\section{Therapie}

Beim unkomplizierten Verlauf einer Samonellose ist keine Antibiotikatherapie nötig, da sie auch zu keiner signifikanten Reduktion der Entwicklung von Dauerausscheidern führt. Da Salmonellosen aber in $2-4 \%$ einen bakteriämischen Verlauf haben, wird bei Patienten mit entsprechender Anamnese (Gefäßimplantate, Gelenksprothesen, degenerative Gelenkserkrankungen, Aneurysmen, Cortisontherapie, Herzklappenerkrankungen, lymphoproliferative Erkrankung, Hämoglobinopathie, Transplantation, AIDS, Krebserkrankung) eine Antibiotikatherapie empfohlen:

- Fluoroquinolone (5-7 Tage: Ciprofloxacin $500 \mathrm{mg} 2 \times$ tgl., Norfloxacin $400 \mathrm{mg}$ $2 \times$ tgl., Levofloxacin $500 \mathrm{mg} 1 \times$ tgl.),

- Trimethoprim-Sulfamethoxazol $(160 / 800 \mathrm{mg}) 2 \times$ tgl. für 5-7 Tage.

Bezüglich S. Typhi und paratyphi wird eine steigende Chinolonresistenz v. a. in Asien beobachtet. Alternativ zu Ciprofloxacin (500 mg $2 \times$ tgl. für 10 Tage p.o.) können Azithromycin $1 \mathrm{~g}$ am 1. Tag, dann $500 \mathrm{mg}$ für 6 Tage oder Ceftriaxon $2 \mathrm{~g} 1 \times \mathrm{tgl}$. i.v. über 14 Tage verwendet werden.

\section{Campylobacter jejuni und $\mathrm{C}$. coli}

Eine Campylobacterinfektion, die zu den häufigsten Ursachen einer akuten infektiösen Diarrhoe gehört, ist charakterisiert durch Fieber, Tenesmen und meist blutiger Diarrhoe. Die Infektionsdosis beginnt bereits bei 500 Bakterien [15]. Die Übertragung erfolgt meist durch kontaminierte Hühnergerichte; C. jejuni kolonisiert viele Masthühner. Die Inkubationszeit liegt zwischen 16 und 48 Stunden (bis 8 Tagen). Die Erkrankung tritt gehäuft im Sommer auf und ist oft auch Reise-assoziiert. Die Diagnose erfolgt mittels Stuhlkultur.

Eine mögliche Komplikation ist die Entwicklung eines Guillain-Barre- Syndroms oder einer reaktiven Arthritis (Reiter-Syndrom. Arthritis + Konjunktivitis + Urethritis).

\section{Therapie}

Campylobacter spp. zeigen eine deutliche Resistenzzunahme gegenüber Ciprofloxacin. Sie liegt in unserem Krankenhaus bei $49 \%$, die Resistenz gegen Clarythromycin liegt bei 8\% (Daten aus dem Resistenzbericht 2006; Hygieneteam/SMZ-Süd, Kaiser Franz Josef Spital, Wien). Makrolide wie Erythromycin oder Clarithromycin reduzieren die Durchfälle, wenn sie innerhalb von 4 Tagen nach Symptombeginn gegeben werden. Immunsupprimierte Patienten oder Patienten mit schwerem prolongiertem Verlauf profitieren von einer Antibiotikatherapie:

- Ciprofloxacin $500 \mathrm{mg} 2 \times \operatorname{tgl} .5$ Tage,

- Azithromycin $500 \mathrm{mg} 1 \times$ tgl. für 3 Tage, Clarithromycin $500 \mathrm{mg} 2 \times \operatorname{tgl}$. für 5 Tage.

\section{Shigella sonnei, Sh. flexneri, Sh. dysenterica}

sind gramnegative Stäbchen und Erreger der bakteriellen Ruhr. Das Reservoir ist der Mensch, die Übertragung erfolgt fäko-oral.

Shigelleninfektionen sind in unseren Regionen meist Reise-assoziiert. Aufgrund der niedrigen Infektionsdosis (10-100 Bakterien) [16] sind sie jedoch potentielle Verursacher von Ausbrüchen in Heimen, Schulen, Kindergärten. Nach einer Inkubationszeit von 1 (-7) Tagen treten Fieber, Erbrechen, Bauchkrämpfe, schleimig-blutige/ eitrige Durchfälle auf, als Ausdruck einer infektiösen Kolitis. Die Symptome werden v.a. durch Shiga-Toxin verursacht sowie durch die Fähigkeit der Shigellen zur Invasion der Kolonschleimhaut. Daraus ergeben sich die Komplikationen: HUS (hämolytischurämisches Syndrom), toxisches Megakolon, Perforation, intestinale Obstruktion; ein postinfektiöses Reitersyndrom bei HLA B27 positiven Patienten ist möglich.

Die Diagnose erfolgt aus der Stuhlkultur.

\section{Therapie}

Die Antibiotikatherapie einer Shigellose führt zur Verkürzung der Durchfalls- und Fieberdauer und reduziert die Ausscheidung des Erregers, wenn sie rasch begonnen wird. Aufgrund der steigenden Resistenz gegen TMP-SMX und Ampicillin sind Chinolonen der Vorzug zu geben: 
- Ciprofloxacin $500 \mathrm{mg} 2 \times$ tgl. für 3 Tage, Levofloxacin $500 \mathrm{mg} 1 \times$ tgl. für 3 Tage.

\section{Yersienia enterocolitica, Y. pseudotuberkulosis}

sind ubiquitär vorkommende gramnegative Bakterien. Die Infektion erfolgt über kontaminiertes Schweinefleisch bzw. Milchprodukte. Die Inkubationszeit beträgt 1-3 Tage. Der Verlauf ist entweder rein enteritisch (wässrige Durchfälle - oft auch mit etwas Blut, Erbrechen, Fieber) oder manifestiert sich wie eine Appendizitis mit Schmerzen im rechten Unterbauch. Die Diagnose erfolgt aus der Stuhlkultur.

Eine Antibiotikatherapie ist meist nicht notwendig, da die Erkrankung nach maximal einer Woche selbstlimitierend ausheilt, bei schweren, systemischen Erkrankungen ist eine Therapie mit Ciprofloxacin $500 \mathrm{mg} 2 \times \operatorname{tgl}$. über 5 Tage möglich.

\section{Escherichia coli}

Die Colibakterien sind Bestandteil der normalen intestinalen Flora. Einige Serotypen sind jedoch pathogen und mit Diarrhoen assoziiert:

$\begin{array}{ll}\text { enterotoxische E. coli } & \text { ETEC } \\ \text { enteropathogene } & \text { EPEC } \\ \text { enteroinvasive } & \text { EIEC } \\ \text { enterohämorrhagische } & \text { EHEC } \\ \text { enteroaggregative } & \text { EaggEC }\end{array}$

Dabei handelt es sich einerseits meist um Erreger einer Reisediarrhoe, andererseits sind sie in Entwicklungsländern häufige Auslöser von Durchfallserkrankungen bei Kindern.

EIEC wirkt vorwiegend im Kolon und produziert ein dysenterieartiges Bild ähnlich der Shigellenruhr.

EHEC besitzen einen spezifischen Virulenzfaktor: sie produzieren ein oder mehrere Shiga-ähnliche Toxine; die meisten EHEC-Stämme gehören zu dieser Serogruppe O157:H7.

Sie verursachen typischerweise eine blutige Diarrhoe ohne (!) Fieber (ohne Invasion). Die Erreger können sowohl fäko-oral als auch von Mensch zu Mensch übertragen werden (Rindfleischprodukte - Hamburger!). Die Inkubationszeit beträgt 3-9 Tage. Die zunehmende Bedeutung von O157:H7 liegt in den Komplikationen:

- hämolytisch - urämisches Syndrom (HUS),

- thrombotisch - thrombozytopenische Purpura (TTP).

Die hämatologischen Komplikationen werden durch Toxin-Wirkung an mucosanahen Endothelien vermittelt.

Diese sind mit hoher Letalität behaftet (5\%) und treten in 10-15\% der Erkrankungen auf. Beim HUS gehen die Bildung von Thrombin (wahrscheinlich durch beschleunigte Thrombogenese) und die Hemmung der Fibrinolyse der Nierenschädigung voraus und dürften die Ursache dieser Schädigung sein [17].

Eine Antibiotikatherapie (TMP-SMX, Chinolone) von O157:H7-Infektionen ist zu vermeiden, da sich gezeigt hat, dass sie die Produktion von Shiga-Toxin induzieren kann und so das Risiko für HUS erhöht [18].

\section{,Lebensmittelvergiftung“6}

Dieser Ausdruck bezeichnet üblicherweise eine akute Gastroenteritis mit folgenden Charakteristika:

- sehr kurze Inkubationszeit: $<6$ h-24 h,

- Klinik: Fieber, Übelkeit und Erbrechen überwiegen gegenüber von Durchfällen,

- kurze Dauer und selbstlimitierend.

Infektionsquelle sind ungekühlt gelagerte, ungenügend gekochte Lebensmittel oder auch erkranktes Küchenpersonal (,eitriger Finger“). Ursächlich sind Toxine von verschiedenen Bakterienstämmen (Bacillus cereus, Staphylokokkus aureus, Clostridium perfringens). Die Toxine sind meist (Ausnahme: hitzestabiles Toxin des B. cereus) durch Kochen inaktivierbar.

Die Therapie besteht in der Gabe von Flüssigkeit (parenteral) \pm Antiemetika.

Bei E.coli-O157:H7-Verdacht keine Antibiotikatherapie

Intoxikation und

Gastroenteritis 
Die Cholera ist eine sekretorische Diarrhoe mit massivem Flüssigkeits- und Elektrolytverlust

\section{Vibrio cholerae}

Vibrio cholerae der Serotypen O1 (klassische und El Tor) und O139 sind die Auslöser der Cholera. Die Übertragung erfolgt durch Trinkwasser und Meeresfrüchte.

Nach einer Inkubationszeit von wenigen Stunden bis zu einer Woche kommt es zu Reiswasser-ähnlichen Stühlen; verursacht wird diese sekretorische Diarrhoe durch das Choleratoxin, das zu einem massiven Flüssigkeitsverlust und Sekretion von Chlorid ins Darmlumen führt (cave: Elektrolytentgleisung, Hypokaliämie, Azidose, hypovolämischer Schock).

Die Diagnose ergibt sich aus der Stuhlmikroskopie: Gramfärbung, Dunkelfeld- und Phasenmikroskopie. Die Kultur auf Spezialmedien dient der Bestätigung.

In der Therapie ist der Rehydratation und dem Elektrolytausgleich der Vorzug zu geben. Die Erkrankung ist innerhalb weniger Tage selbstlimitierend.

Antibiotikatherapie : Doxycyclin $1 \times 300$ mg (cave Phototoxizität) oder Azithromycin $1 \times 1 \mathrm{~g}$ [19] oder Ciprofloxacin $1 \times 1 \mathrm{~g}$ [20].

\section{Ic) Akute Diarrhoe durch Protozoen}

\section{Gardia lamblia}

Der Erreger der Lambliasis wird durch Trinkwasser, Nahrung oder Kontakt übertragen und gehört meist zu den Ursachen einer Reisediarrhoe. Die Durchfälle, die akut oder chronisch verlaufen können, treten nach einer Inkubationszeit von 1-2 Wochen auf. Die Symptome umfassen Blähungen, Übelkeit, Tenesmen vor dem Absetzen des Stuhles; der Stuhl ist weich oder wässrig, faulig riechend, lehmfarben.

Die Diagnose ergibt sich durch den Nachweis von Trophozoiten oder Zysten im Stuhl oder Duodenalaspirat.

Die Therapie besteht in der Gabe von Metronidazol $(3 \times \operatorname{tgl} .250-500 \mathrm{mg}$ für 57 Tage, p.o.).

Die Erkrankung neigt zu Rezidiven.

\section{Entamoeba histolytica, E. dispar}

Die pathogene E. histolytica kann mikroskopisch nicht von der apathogenen E. dispar unterschieden werden. Die Infektion erfolgt durch Aufnahme von Zysten aus kontaminierter Nahrung und Trinkwasser. Die Inkubationszeit beträgt einige Tage bis Monate. Im Darm entwickeln sich aus jeder Zyste acht Trophozoiten, die beweglich und zur Invasion befähigt sind. Die Gewebeform von Entamoeba histolytica hat zytolytische Eigenschaften, die ein Eindringen in die Darmwand und deren Penetration ermöglichen. Extraintestinale Formen zeigen die Fähigkeit Parenchymzellen aufzulösen (Abszessbildung, Leberabszess).

Man unterscheidet:

- asymptomatische Infektion,

- intestinale Verlaufsform mit Fieber, Tenesmen und einer ulzerösen Kolitis,

- extraintestinale Form: v.a. Leberabszess.

Komplikationen der intestinalen Verlaufsform sind: toxisches Megakolon, Peritonitis, Amöbom (intraluminaler Abszess).

Die Diagnose erfolgt durch die Stuhlmikroskopie durch den Nachweis von Zysten oder Trophozoiten. Nur bei invasiven Erkrankungen ergänzt der serologische Nachweis die Diagnose.

Die Therapie besteht in der Gabe von Metronidazol $3 \times$ tgl. 500-750 mg über $7-$ 10 Tage. Zur Eliminierung der Zysten gibt man Paromomycin $30 \mathrm{mg} / \mathrm{KG} / \mathrm{Tag}$ in 3 Dosen über 5-10 Tage oder Diloxanid furoat $500 \mathrm{mg} 3 \times$ tgl. über 10 Tage.

\section{Cyclospora cayetanensis}

hat an Bedeutung gewonnen als Erreger von Durchfallserkrankungen von HIV positiven Patienten und als Erreger von Lebensmittel-bedingten Ausbrüchen [21].

Die Erkrankung beginnt mit leichtem Fieber, Grippe-ähnlichen Symptomen und akuter Diarrhoe. Die Erkrankung verläuft meist selbstlimitierend, kann aber insbesondere bei HIV-positiven Patienten über Wochen und Monate bestehen und zu Müdigkeit und Gewichtsverlust führen. 
Die Diagnose erfolgt mit modifizierter Ziehl-Neelsen-Färbung; die Diagnostik mittels PCR ist nicht allgemein verfügbar.

Therapie

Trimethoprim-Sulfamethoxazol (160/800 mg) $2 \times \operatorname{tgl}$. für 7-10 Tage [22].

\section{Cryptosporidium spp.}

Vorwiegend C. parvum ist beim Menschen die Ursache von Erkrankungen; bei Tieren insbesondere bei Kälbern sind Cryptosporidien weit verbreitet. Oral werden Oozysten aufgenommen, die sich im Dünndarmepithel zu Trophozoiten entwickeln. Nach 3-5 Tagen entwickelt sich eine wässrige Diarrhoe, abdominelle Krämpfe und subfebrile Temperaturen. Bei Immunkompetenten verläuft die Erkrankung selbstlimitierend, bei Immunkompromitierten findet man schwere und längere Verläufe.

Die Diagnose erfolgt durch den Nachweis von Oozysten im angereicherten ZiehlNeelsen gefärbten Stuhl.

\section{Therapie}

Paromomycin \pm Azithromycin verringert lediglich die Symptome; für Nitozoxanid (500 mg $2 \times$ tgl. über 3 Tage) konnte bei Kindern und Immunkompetenten gezeigt werden, dass die Durchfallsdauer verkürzt wird und keine Erreger mehr im Stuhl nachzuweisen waren [23]. Eine 14-tägige Therapie bei Immunsupprimierten erreicht eine $60 \%$ Responserate.

\section{II) Reisediarrhoe}

Viele Reisende in sogenannte Hochrisikogebiete (Asien, Afrika - mit Ausnahme von Südafrika, Süd- und Zentralamerika) entwickeln eine Diarrhoe, deren Symptome schon in den ersten 4 Tagen auftreten können und meist innerhalb von 24 Stunden selbstlimitierend sind. Die „klassische“ Reisediarrhoe dauert länger als 24 Stunden und ist begleitet von Übelkeit, Erbrechen, Bauchkrämpfen, Fieber und blutig/schleimigen Durchfällen.

Ursache können sowohl virale (Noroviren, Rotaviren) als auch bakterielle Erreger und Parasiten (Gardia lamblia, Cryptosporidium parvum, Entamoeba histlytica, Cyclospora cayetanensis) sein. Bakterien sind die jedoch die überwiegenden Verursacher; unter ihnen führend sind ETEC, gefolgt von Salmonella spp., Campylobacter spp., Shigella spp.

In Klinik und Diagnose unterscheidet sich die Reisediarrhoe nicht von der ambulant (außerhalb des Krankenhauses erworbene Diarrhoe).

\section{Therapie der akuten bakteriellen Diarrhoe und der Reisediarrhoe}

1) Rehydratation: Der Flüssigkeits- und Elektrolytersatz, oral oder parenteral, steht an erster Stelle der Therapie.

Die von der WHO empfohlene orale Rehydratationslösung enthält: $90 \mathrm{mmol} \mathrm{Na}$, $20 \mathrm{mmol} \mathrm{K}, 80 \mathrm{mmol} \mathrm{Cl}, 2,5 \mathrm{mmol}$ Bikarbonat, $111 \mathrm{mmol}$ Glukose. Diese Zusammensetzung findet sich in Fertigpräparaten (Pulverform), die Bestandteil der Reiseapotheke sein sollten oder sie kann annähernd erreicht werden durch Mischung von Salz (1 Teelöffel) und Zucker (4 TL) in 11 Wasser plus 1 Glas Orangensaft oder 2 Bananen, was auf Reisen leichter zugänglich ist.

2) Bezüglich der Antibiotikatherapie gilt es auf die Resistenzsituation des Erregers im Reiseland zu achten, z.B. zunehmende Ciprofloxacin-Resistenz, bekannte TMPSMX- und Ampicillinresistenz. In der Behandlung der bakteriellen Diarrhoe soll die Antibiotikatherapie zu einer Verkürzung der Fieber- und Durchfallsdauer führen, sowie die Erreger eliminieren. Diesen Anforderungen stehen Kosten und Resistenzentwicklung entgegen.

3) Motilitätshemmer (Loperamid): durch Verminderung der Darmmotilität und die antisekretorische Wirkung wird die Stuhlfrequenz reduziert und die Bauchkrämpfe sistieren; die Erreger werden dadurch nicht eliminiert. In der Reisemedizin ist Loperamid kontraindiziert bei fieberhafter, blutiger Diarrhoe ohne gleichzeitige Anti-

Epidemiologie der Reisediarrhoe 
Prophylaxe nicht vergessen!

Pathogenese der

Clostridiencolitis biotikatherapie (mögliche Komplikationen: Verlängerung der Abfieberungszeit, toxisches Megacolon, insbes. bei Shigellose).

4) Ernährung/Diät: Bezüglich Diät gibt es keine gesicherten Daten; aufgrund eines möglichen transienten Laktosemangels, sollen Milch und Milchprodukte vermieden werden.

\section{Prophylaxe der Reisediarrhoe}

a) Expositionsprophylaxe

Sie steht an erster Stelle der Maßnahmen und inkludiert alle Vorsichtsmaßnahmen bezüglich Trinkwasser, nicht gegarten Lebensmitteln (Fleisch, Milch), Meeresfrüchten (Muscheln).

\section{b) Chemoprophylaxe}

Der Einsatz von Antibiotika ist aufgrund der möglichen Resistenzentwicklung sehr umstritten. Neben den Kosten ist bei Einsatz von Antibiotika auf Nebenwirkungen wie Phototoxizität (Doxycyclin) und Allergien zu achten. Sie können zu Veränderungen der Darmflora und Kolonisation durch resistente Erreger führen, weiters Pilzinfektionen wie Candidavaginitis begünstigen und schließlich auch eine Clostridiencolitis verursachen.

c) Impfungen

- Choleraimpfung: sie wird von der WHO nicht länger empfohlen, da sie eine geringe Schutzrate aufweist und die Transmission nicht verhindert.

- Impfung gegen Typhus abdominalis: es gibt zwei Formen - eine Schluckimpfung/ Lebendimpfstoff (Vivotif) und ein parenteraler Polysaccharidimpfstoff (Typhim Vi). Die Schluckimpfung soll nicht an Immunsupprimierte oder Personen unter Cortisontherapie verabreicht werden. Die Impfung schützt nicht 100\%ig gegen die Erkrankung, diese verläuft abgeschwächt. Die parenterale Impfung weist einen höheren Impfschutz auf, soll aber ebenfalls nicht dazu führen, dass die Expositionsprophylaxe vernachlässigt wird.

\section{III) Nosokomiale Diarrhoe}

Die beiden häufigsten nosokomialen Durchfallserkrankungen sind:

- Clostridium difficile Enterocolitis,

- Norovirus assoziierte Diarrhoe (vgl. Kapitel Akute virale Diarrhoe).

\section{Clostridium difficile Enterocolitis}

Clostridium difficile ist der Hauptverursacher der sogenannten Antibiotikacolitis. Fast alle Antibiotika können zu dieser Erkrankung führen, v.a. aber Clindamycin, Breitspektrum-Penicilline und Cephalosporine. Die per orale Verabreichung ist mit etwa gleichem Risiko verbunden wie die parenterale, besonders bei Substanzen mit enterohepatalem Kreislauf. Clostridium difficile kolonisiert das Darmlumen, wenn die normale Darmflora durch Antibiotika (auch durch Chemotherapeutika in der Krebstherapie) verändert wurde, was bedeutet, dass ,normalerweise“ einige Organismen/ Gruppen von Organismen diese Kolonisation verhindern. So ist Bacteroides bei vielen Patienten mit C. difficile nicht nachweisbar. Diese Beobachtung führte auch zur sogenannten „Bakteriotherapie“ (z.B. rektale Infusion einer Mischung aus 10 verschiedenen aeroben und anaeroben Bakterien).

Eine Antibiotikacolitis kann auch durch andere Bakterien verursacht werden: C. perfringens Typ A, Candida albicans, S. aureus. Antibiotika selbst können durch andere Mechanismen eine Diarrhoe (mit-)verursachen: Erythromycin wirkt als Motilin-Rezeptor-Agonist und führt so zu beschleunigter Entleerung, Clavulansäure stimuliert die Dünndarmmotilität.

C. difficile produziert zwei Exotoxine (Toxin A und B), die an Rezeptoren des Darmepithels gebunden zu massiver Sekretion und akuter entzündlicher Infiltration führen. Risikofaktoren, eine Antibiotikacolitis zu erwerben sind: 
Alter, Aufenthalt im Krankenhaus (bis zu 30\% der Patienten sind kolonisiert im Gegensatz zu 3\% außerhalb des Krankenhauses) [24]. Dauer des Aufenthaltes, vorausgehende Antibiotikatherapie, Sondenernährung, gastrointestinale Operationen, Kontakt zu einem an C. difficile erkrankten Patienten (Mitpatient im selben Zimmer).

Weiters wurden untersucht:

- der Einfluss der Immunreaktion (kolonisierte Patienten, die einen erhöhten Wert von IgG gegen Toxin A aufwiesen, hatten ein geringeres Risiko eine Diarrhoe zu entwickeln [25], werden eher asymptomatische Träger),

- und genetische Faktoren (genetisch determinierte Fähigkeit, vermehrt IL-8 zu produzieren, ist mit einer Diarrhoeentwicklung assoziiert) [26].

\section{Klinik der Clostridium diffile Enterocolitis}

Sie reicht vom asymptomatischen Träger (scheidet die Erreger aus und stellt so ein ständiges Reservoir dar) bis zur schweren fulminanten Kolitis mit Ausbildung eines toxischen Megakolon. Die Symptome entwickeln sich meist 5-10 Tage nach Antibiotikatherapie (min. 1 Tag - max.10 Wochen). Dazu gehören Fieber, Leukozytose, CRP-Anstieg, Bauchkrämpfe, Nachweis von Leukozyten im Stuhl. Die Diagnose ergibt sich durch Toxinnachweis und Kultur. Eine sofern durchgeführte Endoskopie zeigt das Bild der pseudomembranösen Colitis, in der Computertomographie kann man eine Verdickung der Colonwand sehen.

Therapie der Costridienenterocolitis:

- Metronidazol $500 \mathrm{mg} 3 \times \operatorname{tgl}$. (250 mg $4 \times \operatorname{tgl}$.) für 10 bis 14 Tage,

- Alternativen sind Vancomycin p.o. (4 x 125/250, $3 \times 500 \mathrm{mg}$ tgl.), das jedoch wesentlich teurer ist und das Risiko erhöht für die Entwicklung von Vanco-resistenten Enterokokken,

- weiters Fusidinsäure $(3 \times \operatorname{tgl} .500 \mathrm{mg})$ oder Teicoplanin [27]. Clostridium difficile Enterocolitis rezidiviert in 10-25\%.

Therapie des Rezidivs: Metronidazol - mit einer längeren Therapiedauer (4-6 Wochen) oder Vancomycin in steigender Dosierung.

Weiters untersucht wurden: Saccharomyces boulardii $(250 \mathrm{mg} 2 \times$ tgl.) und Lactobacillus spp., die C. difficile antagonisieren.

\section{Hygienemaßnahmen}

Beide nosokomialen Durchfallserkrankungen sind aufgrund ihrer Übertragbarkeit von großer krankenhaushygienischer Bedeutung.

a) Die Empfehlungen des Robert Koch-Institutes zu Hygienemaßnahmen bei Patienten mit Durchfällen aufgrund von toxinbildendem Clostridium difficile umfassen:

- räumliche Unterbringung: anzustreben ist die Einzelunterbringung in einem Zimmer mit eigener Nasszelle,

- Barrieremaßnahmen: Einsatz von hinsichtlich des Übertragungsweges „Kontakt" geschultem Personals, Verwenden von Schutzkittel und Einweghandschuhen, Schutzmasken, sorgfältige Händehygiene nach direktem Patientenkontakt und nach Kontakt mit Erreger-hältigem Material,

- Desinfektion und Reinigung von Flächen, Aufbereitung von Medizinprodukten,

- Schlussdesinfektion, Abfallentsorgung,

- Transport des Patienten.

b) Die vom Hygieneteam unseres Krankenhauses (SMZ-Süd, Kaiser Franz Josef Spital) empfohlenen Maßnahmen bei Verdacht auf Norovirusinfektion umfassen:

- strenge Isolierung des Patienten bzw. Kohortierung von mehreren betroffenen Patienten,

- keine Neuaufnahmen mit anderen Diagnosen an der betreffenden Station (Bettensperre in Ausbruchsituationen),

- Barrieremaßnahmen, Desinfektion, Aufbereitung von Medizinprodukten, Schlussdesinfektion, Abfallentsorgung, Transport des Patienten (vgl. oben),

Klinik und Therapie der Clostridiencolitis

Nosokomiale Diarrhoe und Hygienemaßnahmen 
- Instruktion der Besucher bezüglich Händedesinfektion, Beschränkung der Besucheranzahl,

- erkranktes Personal bleibt bis 48 Stunden nach Symptomfreiheit im Krankenstand.

\section{Meldepflicht}

Laut Epidemiegesetz 1950 und Änderung mit Bundesgesetz BGB1 I Nr. 114/2006 besteht bei folgenden Durchfallserkrankungen bei Verdachts-, Erkrankungs- und Todesfällen Anzeigepflicht:

Cholera, bakterielle und virale Lebensmittelvergiftungen, Paratyphus, übertragbare (Ruhr), Typhus (Abdominaltyphus).

Epidemiegesetz und Meldepflicht

Jedes Labor, das den Erreger einer meldepflichtigen Erkrankung diagnostiziert, ist zur Erstattung einer Meldung an die zuständige Bezirksverwaltungsbehörde (Gesundheitsamt) binnen 24 Stunden verpflichtet.

Aus den Formularen, die der behandelnde Arzt im Rahmen der Anzeige ausfüllt, gehen die wesentlichen epidemiologischen Daten hervor wie: mögliche Infektionsquelle, Kontaktpersonen in Haushalt, Schule, Arbeitsplatz und inwieweit noch Krankheitserreger ausgeschieden werden (Stuhlkultur).

Die Gesundheitsämter führen dann die Nachkontrollen der Stuhlkulturen durch mit eventueller Einbeziehung der Kontaktpersonen, erteilen sofern erforderlich Arbeitserlaubnis oder nehmen Untersuchungen möglicher Infektionsquellen vor (Restaurants, Lebensmittelproduzenten etc.).

\section{Literatur}

1. Bern C, Martines J, de Zoysa I, Glass RI (1992) The magnitude of the global problem of diarrhoea ldisease; A ten year update. Bull World Health Organ 70: 705-714

2. Herikstad H, Yang S, Van Gilder TJ, Vugia D, Hadler J, Blake P, Deneen V, Shiferaw B, Angulo FJ (2002) A population-based estimate of the burden of diarrhoeal illness in the United States: FoodNet, 1996-7. Epidemiol Infect 129: 9-17

3. Thielman NM, Guerrant RL (2004) Clinical practice. Acute infectious diarrhea. N Engl J Med 350: 38-47

4. Guerrant RL, Araujo V, Soares E (1992) Measurement of fecal lactoferrin as a marker of fecal leukocytes. J Clin Microbiol 30: 1238-1242

5. Guerrant RL, Van Gilder T, Steiner TS, Thielman NM, Slutsker L, Tauxe RV, Hennessy T, Griffin PM, DuPont H, Sack RB, Tarr P, Neill M, Nachamkin I, Reller LB, Osterholm MT, Bennish ML, Pickering LK; Infectious Diseases Society of America (2001) Practice guidelines for the management of infectious diarrhea. Clin Infect Dis 32: 331-351

6. Lopmann B, Reacher MH, van Duijnhoven Y, Hanon FX, Brown B, Koopmans M (2003) Viral Gastroenteritis Outbreaks in Europe, 1995-2000. Emerg Infect Dis 9: 90-96

7. Kaplan JE, Feldmann R, Campbell DS, Lookabaugh C, Gary GW (1982) The frequency of a Norwalk-like pattern of illness in outbreaks of acute gastroenteritis. Am J Public Health 72: $1329-1332$

8. Zahorsky J (1929) Hyperemesis heimis or the winter vomiting disease. Arch Pediatr 46: 391

9. Lopman B, Vennema H, Kohli E, Pothier P, Sanchez A, Negredo A, Buesa J, Schreier E, Reacher M, Brown D, Gray J, Iturriza M, Gallimore C, Bottiger B, Hedlund KO, Torven M, von Bonsdorff CH, Maunula L, Poljsak-Prijatelj M, Zimsek J, Reuter G, Szucs G, Melegh B, Svennson L, van Duijnhoven Y, Koopmans M (2004) Increase in viral gastoenteritis outbreaks in Europe and epidemic spread of new Norovirus variant. Lancet 363: 682-688

10. Anderson EJ, Weber SG (2004) Rotavirus infections in adults. Lancet Infect Dis 4: 91-99

11. Ball JM, Tian P, Zeng CQ, Morris AP, Estes MK (1996) Age-dependent diarrhea induced by a rotaviral nonstructural glycoprotein. Science 272: 101-104

12. Widdowson MA, Bresee JS, Gentsch JR, Glass RI (2005) Rotavirus disease and its prevention. Curr Opin Gastroenterol 21: 26-31

13. Neal KR, Briji SO, Slack RC, Hawkey CJ, Logan RF (1994) Recent treatment with H2 antagonists and gastric surgery as risk factors for Salmonella infection. BMJ 308: 176

14. Parry CM, Hien TT, Dougan G, White NJ, Farrarv JJ (2002) Medical progress: Typhoid fever. N Engl J Med 347: 1770-1782

15. Black RE, Levine MM, Clements ML, Hughes TP, Blaser MJ (1988) Experimental Campylobacter jejuni Infection in humans. J Infect Dis 157: 472-479 
16. Bennish ML (1991) Potentially lethal complications shigellosis. Rev Infect Dis 13 (Suppl 4): S319

17. Chandler WL, Jelacic S, Boster DR, Ciol MA, Williams GD, Watkins SL, Igarashi T, Tarr PI (2002) Prothrombotic coagulation abnormalities preceeding the haemolytic-uremic syndrome. N Engl J Med 346: 23-32

18. Wong CS, Jelacic S, Habeeb RL, Watkins SL, Tarr PI (2000) The risk of the hemolyticuremic syndrome after antibiotic treatment of Escherichia coli O157:H7 infections. N Engl J Med 342: 1930-1936

19. Saha D, Karim MM, Khan WA, Ahmed S, Salam MA, Bennish ML (2006) Single-dose Azithromycin fo the treatment of cholera in adults. N Engl J Med 354: 2452-2462

20. Khan WA, Bennish ML, Seas C, Khan EH, Ronan A, Dhar U, Busch W, Salam MA (1996) Randomised controlled comparison of single-dose ciprofloxacin and doxycycline for cholera caused by Vibrio cholerae O1 or O139. Lancet 348: 296-300

21. Herwaldt BL (2000) Cyclospora cayetanensis: a review, focusing on the outbreaks of cyclosporiasis in the 1990s. Clin Infect Dis 31: 1040-1057

22. Hoge CW, Shlim DR, Ghimire M, Rabold JG, Pandey P, Walch A, Rajah R, Gaudio P, Echeverria P (1995) Placebo-contolled trial of co-trimoxazole for Cyclospora infections among travellers and foreign residents in Nepal. Lancet 345: 691-693

23. Rossignol JF, Ayoub A, AyersMS (2001) Treatment of diarrhea caused by Cryptosporidium parvum: a prospective randomised, double-blind, placebo-controlled study of nitazoxanide. J Infect Dis 184: 103-106

24. Bartlett JG (2002) Antibiotic-associated diarrhea. N Engl J Med 346: 334-339

25. Kyne L, Warny M, Qamar A, Kelly CP (2000) Asymptomatic carriage of Clostridium difficile and srum levels of IgG antibody against toxin A. N Engl J Med 342: 390-397

26. Jiang ZD, DuPont HL, Garey K, Price M, Graham G, Okhuysen P, Dao-Tran T, LaRocco M (2006) A common polymorphism in the Interleukin 8 Gene Promotor is associated with Clostridium difficile diarrhea. Am J Gastroenterol 101: 1112-116

27. Wenisch C, Parschalk B, Hasenhundl M, Hirschl AM, Graninger W (1996) Comparison of vancomcin, teicoplanin, metronidazole and fusidic acid fort he treatment of Clodridium difficile-associated diarrhea. Clin Infect Dis 23: 423

Korrespondenz: OA Dr. Gertrude Seiberl, 4. Medizinische Abteilung mit Infektions- und Tropenmedizin, SMZ-Süd, Kaiser Franz Josef Spital, Kundratstrasse 3, 1100 Wien, Österreich, E-mail: gertrude.seiberl@wienkav.at 


\section{Vorbereitungsfragen zur Facharztprüfung}

120. Die häufigsten viralen Erreger von Gastroenteritiden bei Erwachsenen weltweit sind

a) Noroviren

b) Rotaviren

c) Adenoviren

d) Astroviren

121. In einem Altersheim kommt es bei den Bewohnern zu einem Gastroenteritisausbruch mit akut einsetzendem starken Erbrechen und Durchfall. Die Krankheitsdauer ist durchschnittlich 2 Tage

a) Die wahrscheinlichsten Erreger sind in diesem Fall Salmonellen.

b) Die wahrscheinlichsten Erreger sind in diesem Fall Noroviren.

c) Die Weiterverbreitung der Keime kann nur fäko-oral erfolgen

d) Aufgrund des Alters der Patienten sollte sofort eine antibiotische Therapie erfolgen

122. Die Methode der Wahl zum Nachweis von Noroviren ist

a) Virusnachweis mittels ELISA aus dem Stuhl

b) Elektronenmikroskopischer Nachweis aus dem Stuh

c) Kultureller Nachweis aus dem Stuhl

d) Virusnachweis mittels RT-PCR aus dem Stuhl

123. In einem Kindergarten erkranken innerhalb von 2 Tagen 6 Kinder im Alter von 1 bis 2 Jahren an Erbrechen und Durchfall. Zusätzlich leiden die Kinder an Husten und Schnupfen

a) Es besteht der Verdacht auf Shigelleninfektion. Die erkrankten Kinder sollten umgehend eine antibiotische Therapie erhalten.

b) In dieser Symptomkonstellation ist die virale Genese (Rotaviren) des Durchfalls am wahrscheinlichsten Die wichtigste Maßnahme ist ausreichende Flüssigkeitszufuhr.

c) Sobald die erkrankten Kinder symptomfrei sind, können sie wieder den Kindergarten besuchen.

d) Eine Infektionsgefahr für Erwachsene besteht nicht.

124. Ein Patient leidet unter blutiger Diarrhoe, Fieber bis $\mathbf{3 8}^{\circ}$ und krampfartigen Bauchschmerzen. Zwei Tage zuvor hatte auf dem Flug von Miami nach Wien eine Geflügelmayonnaise gegessen. Welcher Durchfallserreger kann für die Symptomatik in Frage kommen?
a) Campylobacter
b) Cyclospora
c) E. coli
d) Entamoeba histolytica

125. Ein Patient, der vor 3 Jahren eine Hüftendoprothese links implantiert bekam, leidet an einer akuten Salmonellenerkrankung mit Fieber von $38,5^{\circ}$, Erbrechen, Hypokaliämie, CRP 320 U/ml. Die Stuhlkultur war positiv, die Blutkultur negativ. Welche therapeutischen Maßnahmen werden gesetzt?

a) Orale Rehydratation

b) Parenterale Rehydratation plus Antiemetikum

c) Orale Rehydratation plus p.o. Antibiotikum

d) B plus Antibiotikum, anfänglich i.v. dann p.o.

126. Ein Patient, der von einem mehrmonatigen Aufenthalt im Nahen Osten zurückkehrt, leidet seit 2 Wochen an Flatulenz, übelriechender und lehmfarbener Diarrhoe. Welche diagnostischen Maßnahmen sind zielführend?

a) Stuhlkultur

b) Stuhlmikroskopie auf Leucozyten und Erythrozyten

c) Parasitologische Stuhluntersuchung

d) Gastroskopie mit Aspiration von Duodenalsaft

127. Sie werden gebeten eine

Reiseberatung bezüglich Durchfallserkrankungen durchzuführen; die Reise ist eine Kombination aus Rundreise im Jeep und einem anschließenden Badeaufenthalt in Kenia. Welche Empfehlungen geben sie?

a) Erklärungen zur Expositionsprohylaxe

b) Einnahme von Loperamid bis zum Sistieren der Durchfälle

c) Täglich ein Ciprofloxacin $500 \mathrm{mg}$

d) Mitnahme einer Elektrolytlösung in Pulverform und eines Antbiotikums für den Fall einer fieberhaften, blutig-schleimigen Diarrhoe

128. Eine Patientin erhält wegen einer Furunkulose eine 7 Tage Therapie mit $3 \times 600 \mathrm{mg}$ Clindamycin p.o. Eine Woche später treten Bauchkrämpfe, Fieber und eine Diarrhoe auf. Nach der Diagnose einer Clostridium difficile Enterocolitis erhält die Patientin Metronidazol $3 \times 500 \mathrm{mg}$ tgl. über 7 Tage. Nach einem symptomfreien Intervall treten erneut Fieber und Diarrhoe auf. Welche diagnostischen Maßnahmen setzen Sie zuerst?

a) Stuhlmikroskopie

b) Stuhlkultur inkl. Nachweis von Toxin A

c) Coloskopie

d) $\mathrm{A}+\mathrm{B}$ und $\mathrm{CT}-\mathrm{Abdomen}$

129. Welche Therapie führen Sie durch?

a) Metronidazol $3 \times 500 \mathrm{mg}$ tgl. für 7 Tage

b) Metronidazol $3 \times 500 \mathrm{mg}$ tgl. für 14 Tage in Kombination mit Saccharomyces boulardii $2 \times$ $250 \mathrm{mg} \operatorname{tgl}$

c) Vancomycin i.v.

d) Vancomycin $4 \times 125 \mathrm{mg}$ tgl. für 5 Tage

130. Ein junger Mann erkrankt nach einem Essen in einem Restaurant an einer blutigen Diarrhoe ohne Fieber. In der Stuhlmikroskopie findet man keinen Hinweis auf das Vorliegen einer invasiven Diarrhoe. Welche Therapie würden Sie wählen?

a) Der Patient erhält eine parenterale Flüssigkeitssubstitution und Ciprofloxacin p.o. wegen Verdacht auf Salmonelleninfektion.

b) Wegen Verdacht auf Campylobacteriose erhält er ein Makrolid p.o.

c) Es wird eine p.o.Rehydratation durchgeführt und kein Antibiotikum gegeben, da eine Infektion durch E.coli nicht ausgeschlossen werden kann.

d) Der Gyrasehemmer und das Makrolid werden kombiniert.

Die Lösung zu dieser Fortbildungseinheit erfahren Sie in der nächsten bzw. übernächsten Ausgabe. 